\title{
Computed Tomography Images of the Scapula Taken With Reduced Dose Can Yield Segmented Models of Sufficient Accuracy: A Pilot Study
}

\author{
Michiel Herteleer, MD, * Sanne Vancleef, MSc, † Stefaan Nijs, MD, PhD, *+ and Jos Vander Sloten, PhD $†$
}

\begin{abstract}
Objective: The aim of the study was to investigate the influence of tube current reduction on the segmentation accuracy of the scapula.

Methods: A human cadaver was computed tomography scanned multiple times while reducing tube current amperage. The images were segmented using 2 different segmentation methods $(\mathrm{N}=28)$. Subsequently the scapula was dissected and all soft tissues were removed. An optical laser scan of the dissected scapula was aligned and compared with the segmented meshes of the different computed tomography scans.

Results: The mesh accuracy remained fairly constant with diminishing tube currents. All segmented meshes had a larger volume than the reference mesh $(n=27)$. The mean 3-dimensional deviation varied between $1.17 \mathrm{~mm}$ ( $\mathrm{max}$ ) and $-0.759 \mathrm{~mm}(\mathrm{~min})$ and the total mean $(\mathrm{SD}) 3$-dimensional deviation was $-0.45(0.38) \mathrm{mm}$. Radiation dosages were reduced from 7.1 to $0.3 \mathrm{mSv}$. Conclusions: Computed tomography tube current can be largely reduced without losing the surface segmentation accuracy of segmented scapula meshes.
\end{abstract}

Key Words: scapula, segmentation, low-dose CT, 3D

(J Comput Assist Tomogr 2018;00: 00-00)

$T$ he use of computed tomography (CT) scans has become an indispensable part in the diagnostic and preoperative assessment of orthopedic and trauma patients. These CT images are used more and more to make 3-dimensional (3D) reconstructions, aiding the physician in assessing the anatomy and pathology, and for the design of custom-made orthopedic implants. Commercial software packages allow surgeons and engineers to segment these images and to use the resulting $3 \mathrm{D}$ models to improve patient care and research quality. ${ }^{1-3}$ However, there is a downside regarding the increased use of CT scans. The ionizing radiation dosage of $1 \mathrm{CT}$ scan of the shoulder relates to more or less 25 chest x-rays of the thorax. ${ }^{4}$ Orthopedic and trauma patients are often young and could therefore benefit more from a radiation reduction in their musculoskeletal diagnostic work-up.

Several studies have inspected the quality and accuracy of image segmentation modules for long bone structures. They demonstrated a high segmentation accuracy for musculoskeletal scans regarding the tibia and femur. ${ }^{5,6}$ Kwon et al ${ }^{7}$ demonstrated that the distances and angles measured on 3D reconstructed models of scapula images show high interobserver and intraobserver variability rates.

To our knowledge, there are no studies that have investigated the influence of CT dose reduction on the image quality and

From the *Organ Systems, KU Leuven, Leuven; $\uparrow$ Department of Mechanical Engineering, Division Biomechanical Engineering, Heverlee; and $\ddagger$ Traumatology, UZ Leuven, Leuven, Belgium.

Received for publication June 27, 2017; accepted October 24, 2017.

Correspondence to: Michiel Herteleer, MD, Traumatology Herestraat 49, 3000

Leuven, Vlaams-Brabant, Belgium (e-mail:

michiel.herteleer@kuleuven.be).

The authors declare no conflict of interest.

The study was approved by Ethical Committee KU Leuven.

Copyright (C) 2018 Wolters Kluwer Health, Inc. All rights reserved.

DOI: $10.1097 /$ RCT.0000000000000706 especially on how the dose reduction influences the segmentation quality of the scapula.

\section{MATERIALS AND METHODS}

After obtaining approval from the local ethical committee (Ethisch Committee, KU Leuven, Belgium), we obtained 1 fresh frozen cadaver from the human body donation program of the KU Leuven. A standardized axial CT shoulder was obtained on a clinical CT scan, Siemens Somatom Force (Siemens AG, Erlangen, Germany), dual-source/dual-energy, 192-slice. The scan covered the entire glenohumeral joint, and the scan length was determined by a radiological assistant as if it were a normal patient. While maintaining all $\mathrm{CT}$ scan settings, the tube current was reduced gradually until the lowest current at which the CT scan would function was obtained (Table 1). In total, 7 CT scans were obtained from the same cadaveric specimen.

The right scapula was dissected, cleaned manually, and then boiled to remove the remaining fat. It was then optically scanned with the Nikon LC60Dx (Nikon Metrology, United Kingdom) mounted on a coordinate measuring machine (MC16, Coord3, S.p.A, Turin, Italy). The manufacturer reports a surface accuracy between 6 and 36 microns. The obtained point cloud was reconstructed into an STL file using Focus inspect (Nikon Metrology) and then imported into 3-matic (3-matic Research 11.0; Materialise NV, Leuven, Belgium) where a mesh repair was performed. Mesh abnormalities were repaired using different fix methods such as remove overlapping triangles, invert normal, and fill holes.

The obtained CT scans were imported into Mimics Research 19.0 (Materialise NV) and segmented by 2 researchers each using a different protocol. The 2 different segmentation protocols were applied to images with bone and with a soft tissue window, resulting in 4 surface meshes per CT scan.

Michiel Herteleer used an automated segmentation protocol where a standard bone mask threshold (226-3071) was applied to the axial CT images. Then, a region growing was performed to isolate the scapula. The $3 \mathrm{D}$ models were calculated from the masks using the high-quality segmentation settings. The resulting STL file was then imported in to 3-matic (3-matic Research 11.0, Materialise NV) and a wrap operation with a gap closing distance of $4 \mathrm{~mm}$, smallest detail of $0.1 \mathrm{~mm}$, protection of thin walls, and a resulting offset of $0.07 \mathrm{~mm}$ was performed. This was done to create a full and uniform shell. Where necessary, manual fixing methods were applied to create a single shell mesh.

Sanne Vancleef used a semiautomated segmentation method, which can be found as the CT-bone module. A point on the cortex of the scapula was selected on the CT scan and then automatically filled using the automated smart expand and bone filling module. The 3D models were calculated from the masks using the highquality segmentation settings and the resulting STL file was then imported in to 3-matic (3-matic Research 11.0, Materialise NV) without additional wrapping. No wrap operation was applied because the semiautomated segmentation method produces meshes without inner surfaces. ${ }^{6}$ 
TABLE 1. Computed Tomography Scan Settings

\begin{tabular}{|c|c|c|c|c|c|c|c|c|c|}
\hline CT & $\mathbf{k V}$ & mAs & Ref & CTDI, mGy & DLP, mGy.cm & Scan Length, cm & Pitch & ST, mm & Pixel, mm \\
\hline 1 & 110 & 408 & 516 & 21.37 & 379.1 & 18 & 0.8 & 0.6 & $0.85 \times 0.85$ \\
\hline 2 & 110 & 266 & 326 & 13.92 & 246.9 & 18 & 0.8 & 0.6 & $0.85 \times 0.85$ \\
\hline 3 & 110 & 196 & 246 & 10.26 & 182 & 18 & 0.8 & 0.6 & $0.85 \times 0.85$ \\
\hline 4 & 110 & 136 & 167 & 7.12 & 126.4 & 18 & 0.8 & 0.6 & $0.85 \times 0.85$ \\
\hline 5 & 110 & 75 & 87 & 3.92 & 69.6 & 18 & 0.8 & 0.6 & $0.85 \times 0.85$ \\
\hline 6 & 110 & 43 & 46 & 2.29 & 40.6 & 18 & 0.8 & 0.6 & $0.85 \times 0.85$ \\
\hline 7 & 110 & 18 & 18 & 1.02 & 19.4 & 18 & 1.05 & 0.6 & $0.85 \times 0.85$ \\
\hline
\end{tabular}

CTDI indicates computed tomography dose index; DLP, dose-length product.

The meshes were aligned with the optically obtained scapula using a best-fit method. A signed global part comparison analysis was used to determine the $3 \mathrm{D}$ distance between the reference mesh and the segmented mesh. The distance values were imported into GraphPad Prism (Version 7.00 for Windows, GraphPad Software, La Jolla, Calif).

\section{Statistics}

Data were analyzed using Graphpad PRISM. Descriptive statistics were calculated based on frequency tables and histograms were plotted as line graphs. The mean deviations were compared using a one-way analysis of variance test. When the comparison was limited to 2 groups, a $t$ test was used.

\section{RESULTS}

\section{Mesh Difference}

All meshes were statistically significantly different from the optically scanned mesh (ground truth) using a one-way analysis of variance test with multiple comparisons $(P<0.0001)$. All segmented

\section{MH B}

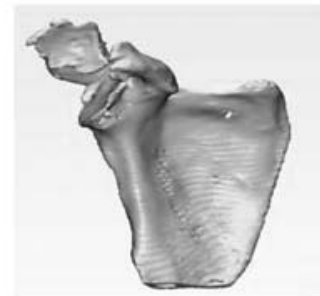

4
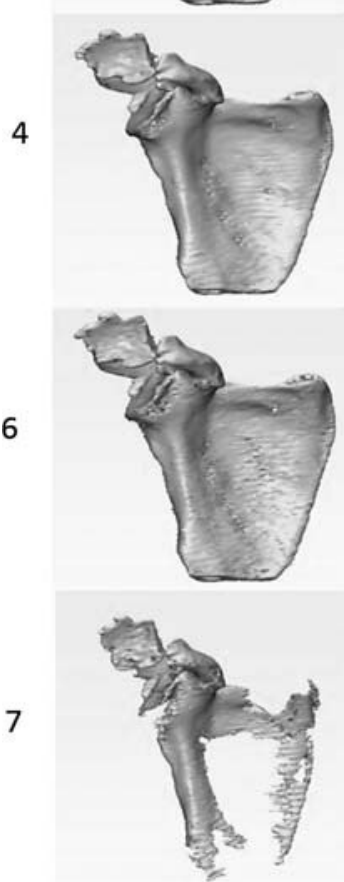

SVC B
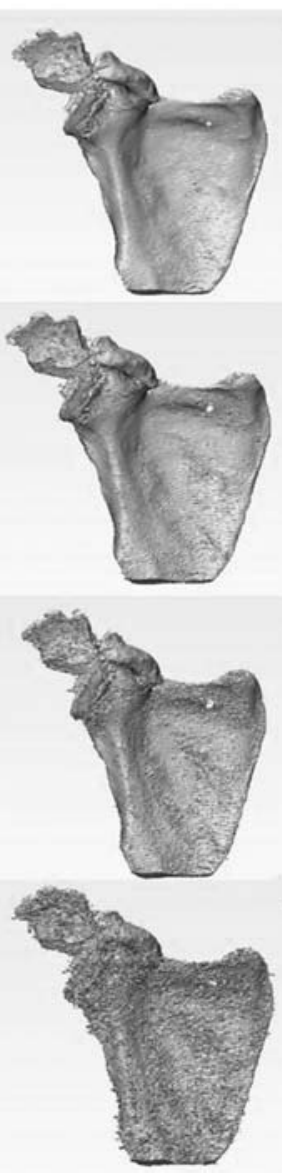

MH ST
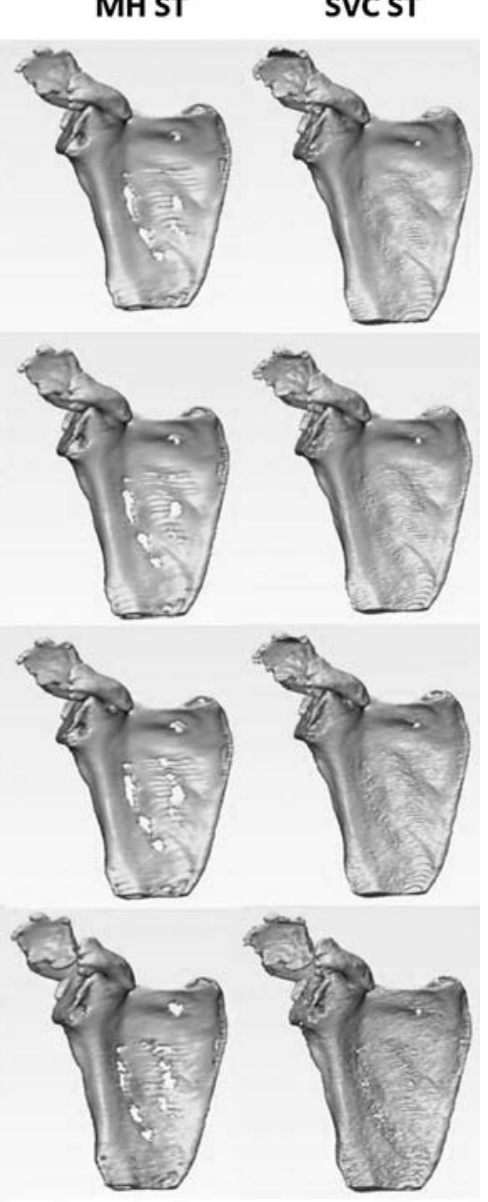

FIGURE 1. Illustration of some of the segmented CT scans. A visual analysis shows that there are no large deformations when the radiation dosage is reduced. Sanne Vancleef 1B provided the highest surface accuracy, which is demonstrated in Figure 2. The automated segmentation by Michiel Herteleer 7B shows a clear volume loss in the body of the scapula. The visually perceived surface roughness of Sanne Vancleef 7B is remarkably larger than that of the other surface meshes. 
TABLE 2. Radiation Dosages of the CT Scans

\begin{tabular}{lcc}
\hline CT & Effective Dose Man, mSv & Effective Dose Woman, mSv \\
\hline 1 & 6.9 & 7.3 \\
2 & 4.5 & 4.8 \\
3 & 3.3 & 3.5 \\
4 & 2.3 & 2.4 \\
5 & 1.3 & 1.3 \\
6 & 0.7 & 0.8 \\
7 & 0.3 & 0.3 \\
\hline
\end{tabular}

meshes, apart from Michiel Herteleer 7B, had larger volumes than the optically scanned reference meshes. This under sizing is due to a volume loss, which is a consequence of the initial segmentation (Fig. 1).

\section{Bone Window vs Soft Tissue Window}

The 3D deviations of all meshes segmented from the CT Images in the soft tissue window were statistically significantly different from meshes segmented from the bone window $(P<0.001)$. From a visual point of view, there seemed to be an increased amount of noise on the surface meshes with regard to lower radiation dose. This noise was more prominent in the models, which were segmented in the bone window than in the models segmented in the soft tissue window (Fig. 1).

\section{Radiation Dosage and Surface Accuracy}

The radiation dosage was computed by a medical physicist based on the scan parameters and the scanned volume (Table 1). Dosages were corrected for male and female cadavers because of the close proximity of the breast region. As expected, the effective dose decreased linearly with decreasing tube currents. However, the mean deviation of our segmented models did not decrease linearly but remained fairly constant apart from the segmentations of the lowest-dosage images (Michiel Herteleer) (Table 2, Fig. 2). ${ }^{8}$

\section{Mesh Volume}

All segmented meshes were significantly larger than the optically scanned reference mesh $(P<0.0001)$. The mean volumes between the meshes by Michiel Herteleer and Sanne Vancleef were not statistically significantly different $(P=0.7594)$. The meshes

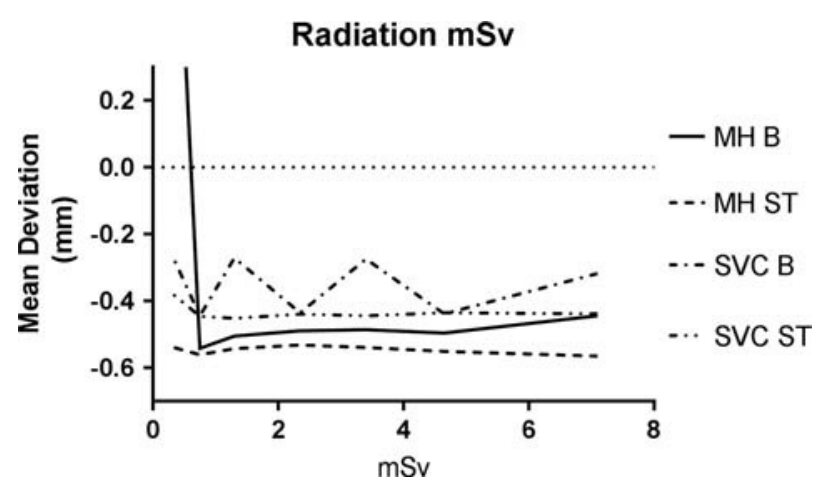

FIGURE 2. Influence of the radiation dosage on the mean deviation of the surface meshes. The mean deviation of the models segmented in the soft tissue window is larger than those segmented in the bone window. The mean deviation of the meshes segmented in the soft tissue window is less influenced by the lowest radiation dosage.

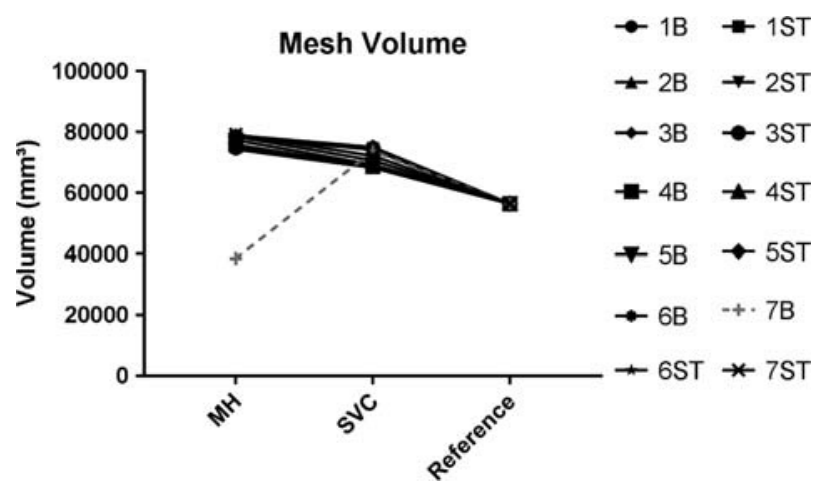

FIGURE 3. All meshes (apart Michiel Herteleer 7B) have larger volumes than the optically laser scanned reference mesh. The fully automated segmented mesh from the lowest-dosage bone window scan (7B) has a largely reduced volume. This can also be clearly seen in Figure 1.

based on the automatic segmentation protocol (Michiel Herteleer) tended to have larger volumes than the meshes created by the semiautomatic CT-bone module (Sanne Vancleef) (Fig. 3).

\section{General Comparison of the Surface Meshes}

The mean 3D distance of the meshes created using the automated segmentation protocol (Michiel Herteleer) varied between -0.44 and $1.17 \mathrm{~mm}$ for the bone window and -0.54 and $-0.57 \mathrm{~mm}$ for the soft tissue window (Table 3, Fig. 4).

The mean 3D distance of the meshes created using the semiautomated segmentation protocol (Sanne Vancleef) varied between -0.44 and $-0.27 \mathrm{~mm}$ for the bone window and -0.48 and $-0.38 \mathrm{~mm}$ for the soft tissue window (Table 4, Fig. 5).

\section{DISCUSSION}

In this pilot study, we investigated the influence of reducing the CT tube current and concomitant radiation dose on the quality of segmented meshes of the scapula. Our research suggests that tube current and therefore radiation dose could be largely reduced without reducing the accuracy meshes of the bone.

TABLE 3. Deviation From the Optical Mesh (Automatically Segmented Meshes)

\begin{tabular}{cccccc}
\hline CT & Mean, mm & SD, $\mathbf{m m}$ & Max, mm & Min, mm & $\mathbf{n}$ \\
\hline \multicolumn{7}{l}{ Michiel } & Herteleer bone & window & & & \\
1B & -0.44 & 0.70 & 2.85 & -2.45 & 28,261 \\
2B & -0.50 & 0.71 & 3.16 & -2.65 & 28,261 \\
3B & -0.49 & 0.73 & 3.14 & -2.62 & 28,261 \\
4B & -0.49 & 0.73 & 3.01 & -2.71 & 28,261 \\
5B & -0.51 & 0.76 & 2.99 & -2.68 & 28,261 \\
6B & -0.54 & 0.76 & 3.08 & -2.57 & 28,261 \\
7B & 1.17 & 2.90 & 19.18 & -1.48 & 28,261 \\
Michiel Herteleer soft tissue window & & & \\
1ST & -0.57 & 0.91 & 3.37 & -3.32 & 28,261 \\
2ST & -0.55 & 0.92 & 3.13 & -3.18 & 28,261 \\
3ST & -0.54 & 0.94 & 3.10 & -3.20 & 28,261 \\
4ST & -0.53 & 0.94 & 3.12 & -3.22 & 28,261 \\
5ST & -0.54 & 0.94 & 3.14 & -3.23 & 28,261 \\
6ST & -0.56 & 0.95 & 3.23 & -3.50 & 28,261 \\
7ST & -0.54 & 0.95 & 3.15 & -3.39 & 28,261 \\
\hline
\end{tabular}



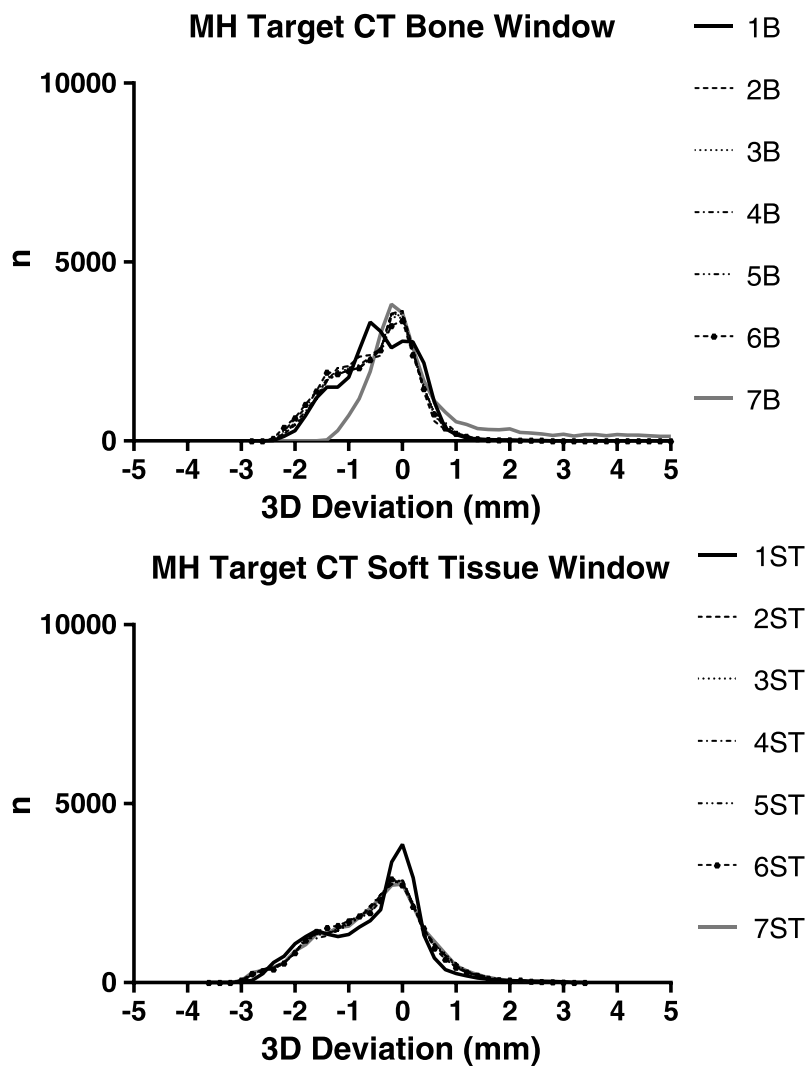

FIGURE 4. The graphs in Figure 4 show the distribution of the deviation for the semiautomatically generated meshes (Michiel Herteleer). The distribution of the curves is similar although there clearly is a visual difference between Michiel Herteleer 7B and the other meshes. This is due to the fact that the calculations are based on the amount of triangles of the meshes. The part of the body of the scapula, which has disappeared (Fig. 1) has only a small amount of triangles compared with more complex shapes such as the acromion. The extrema and the width of the graph give a good appreciation on the general quality of the mesh (Table 3).

TABLE 4. Deviation From the Optical Mesh (Semiautomatically Segmented Meshes)

\begin{tabular}{|c|c|c|c|c|c|}
\hline CT & Mean, mm & SD, $\mathbf{m m}$ & Max, mm & Min, mm & $\mathbf{n}$ \\
\hline \multicolumn{6}{|c|}{ Sanne Vancleef bone window } \\
\hline 1B & -0.32 & 0.41 & 3.05 & -3.83 & 30,790 \\
\hline $2 \mathrm{~B}$ & -0.44 & 0.64 & 2.99 & -3.95 & 30,790 \\
\hline $3 \mathrm{~B}$ & -0.27 & 0.53 & 3.60 & -2.40 & 30,790 \\
\hline $4 \mathrm{~B}$ & -0.44 & 0.71 & 3.72 & -3.20 & 30,790 \\
\hline $5 \mathrm{~B}$ & -0.27 & 0.54 & 3.36 & -3.16 & 30,790 \\
\hline $6 \mathrm{~B}$ & -0.44 & 0.69 & 3.63 & -3.19 & 30,790 \\
\hline 7B & -0.26 & 0.53 & 3.34 & -2.76 & 30,790 \\
\hline \multicolumn{6}{|c|}{ Soft tissue window } \\
\hline $1 \mathrm{ST}$ & -0.44 & 0.64 & 2.99 & -3.95 & 30,790 \\
\hline $2 \mathrm{ST}$ & -0.44 & 0.71 & 3.72 & -3.20 & 30,790 \\
\hline $3 \mathrm{ST}$ & -0.44 & 0.69 & 3.63 & -3.19 & 30,790 \\
\hline $4 \mathrm{ST}$ & -0.44 & 0.69 & 3.63 & -2.79 & 30,790 \\
\hline $5 \mathrm{ST}$ & -0.45 & 0.70 & 3.73 & -3.36 & 30,790 \\
\hline $6 \mathrm{ST}$ & -0.45 & 0.72 & 3.66 & -3.23 & 30,790 \\
\hline $7 \mathrm{ST}$ & -0.38 & 0.79 & 3.31 & -2.51 & 30,790 \\
\hline
\end{tabular}

SVC Target CT Bone Window

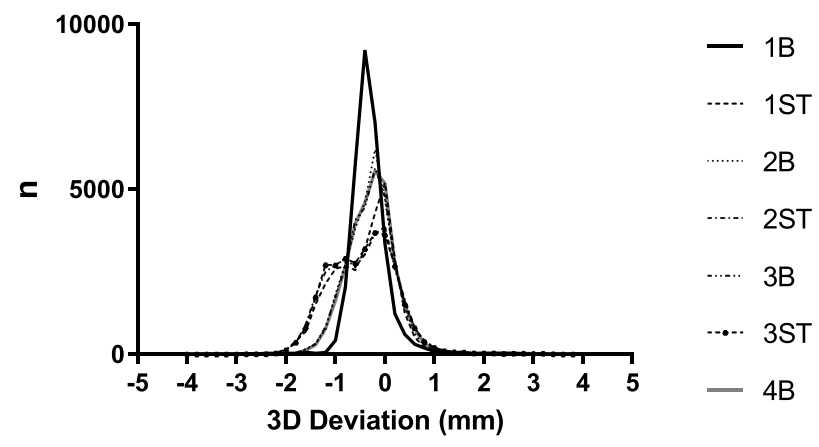

SVC Target CT Soft Tissue Window

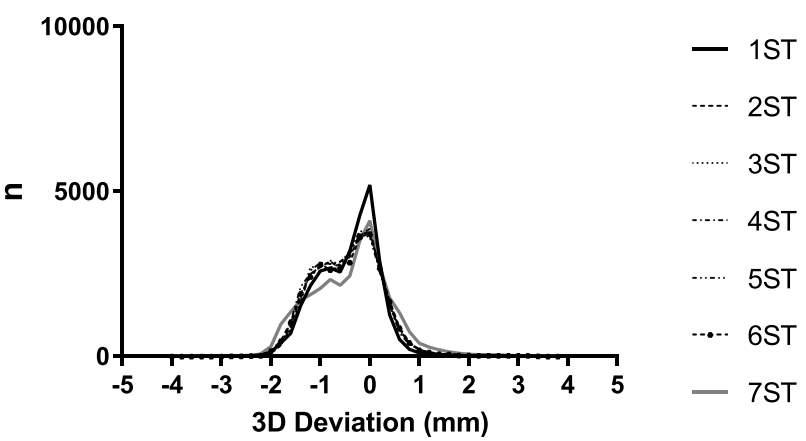

FIGURE 5. The graphs in Figure 5 show the distribution of the deviation for the semiautomatically generated meshes (Sanne Vancleef). The mesh generated from the images in the bone window with the highest radiation dosage (Sanne Vancleef 1B) have the narrowest graph. Although the mean deviation is closer to zero in other graphs, the width of the graph gives a better approximation of the accuracy of the graph.

Our results confirm the results of previous studies, which have proven that long bones can be accurately segmented using CT and MRI images. Although we included only 1 cadaver in our analysis, our results lie in the line of previous studies. They also confirm that CT segmented images overestimate bone geometry but still provide sufficient geometrical information. ${ }^{5,6}$

These previous studies reported on the segmentation accuracy of long bones such as the tibia and femur. This study, however, is the first to our knowledge, which reports on the segmentation accuracy of the scapula, which is a bone with unique characteristics. Its body is very thin walled, and the general shape is more complex and could therefore be more susceptible to segmentation errors.

The surface roughness of the segmented meshes is a parameter, which is not commonly used to describe 3D models. The authors have the impression that the surface roughness of the meshes increased with the use of the semiautomated CT-bone module, especially for models segmented from images obtained from the bone window. To our knowledge, the surface roughness is a parameter, which is difficult to be determined, using a generic method, to describe the general roughness of a virtual 3D surface (STL file).

Furthermore, the used CT scanner and its concomitant software could largely influence the quality of the axial images and therefore the quality of the segmentation.

Future studies could focus on the difference between commercially and freely available software packages. The software package we used (Mimics Research 19.0, Materialise NV) was available in our institution but is not freely available to all researchers. The authors believe that freely available software packages might provide 
an accuracy, which is good enough to allow surgeons to experiment with the $3 \mathrm{D}$ models of their patients before they perform surgery on the patient. Future research should focus on these differences as well.

Most importantly, future research should focus on reducing the radiation exposure while still providing accurate $3 \mathrm{D}$ reconstruction images. This would allow orthopedic and trauma surgeons to use CT imaging more frequently aiding in the design of personalized implants and guides without exposing the patient to unnecessary ionizing radiation.

\section{CONCLUSIONS}

This pilot study confirms the results that $3 \mathrm{D}$ reconstructed CT images provide accurate information of the bone surface. It suggests that this is not only accurate for long bones but also for bones with a more complex shape such as the scapula. The most important conclusion of this pilot study is that a linear reduction of ionizing radiation does not result in a linear decrease of mesh surface quality.

\section{ACKNOWLEDGMENTS}

The authors thank the Vesalius Instituut of the KU Leuven and especially Dr. Paul Herijgers for their continuous work with the human body donation program. The authors also thank Walter Coudyzer and Joke Binst of the UZ Leuven Radiology Department for technical support in data collection. This research was made possible by and IDO grant of the KU Leuven.

\section{REFERENCES}

1. Upex P, Jouffroy P, Riouallon G. Application of 3D printing for treating fractures of both columns of the acetabulum: benefit of pre-contouring plates on the mirrored healthy pelvis. Orthop Traumatol Surg Res. 2017;103: $331-334$.

2. Hoekstra H, Rosseels W, Sermon A, et al. Corrective limb osteotomy using patient specific 3D-printed guides: a technical note. Injury. 2016;47: 2375-2380.

3. Van Tongel A, Huysmans T, Amit B, et al. Evaluation of prominence of straight plates and precontoured clavicle plates using automated plate-to-bone alignment. Acta Orthop Belg. 2014;80:301-308.

4. Biswas D, Bible JE, Bohan M, et al. Radiation exposure from musculoskeletal computerized tomographic scans. J Bone Joint Surg Am. 2009;91:1882-1889.

5. Van den Broeck J, Vereecke E, Wirix-Speetjens R, et al. Segmentation accuracy of long bones. Med Eng Phys. 2014;36:949-953.

6. Gelaude F, Vander Sloten J, Lauwers B. Accuracy assessment of CT-based outer surface femur meshes. Comput Aided Surg. 2008;13: 188-199.

7. Kwon YW, Powell KA, Yum JK, et al. Use of three-dimensional computed tomography for the analysis of the glenoid anatomy. J Shoulder Elb Surg. 2005; 14:85-90.

8. McNitt-Gray MF. AAPM/RSNA Physics Tutorial for Residents: topics in CT. Radiation dose in CT. Radiographics. 2002;22:1541-1553. 